\title{
AVALIAÇÃO DO ESTADO NUTRICIONAL DE PACIENTES ADMITIDOS NO SERVIÇO DE ACOLHIMENTO E CLASSIFICAÇÃO DE RISCO NO HOSPITAL REGIONAL DE PRESIDENTE PRUDENTE - SP
}

\author{
Marcela de Andrade Bernal Fagiani ${ }^{1}$, Sandra Cristina Genaro ${ }^{1,2}$. \\ ${ }^{1}$ Universidade do Oeste Paulista - UNOESTE, ${ }^{1}$ Residência Multiprofissional em Urgência e Trauma, ${ }^{2}$ Curso de Nutrição. \\ E-mail: marcelafagiani16@gmail.com
}

\section{RESUMO}

A desnutrição em ambiente hospitalar é um problema de saúde pública que está associada ao aumento significativo de morbidade e mortalidade. O objetivo do trabalho foi avaliar o estado nutricional por meio de triagem nutricional, adaptada de Malnutrition Screening Tool (MST). Estudo de campo, realizado no Hospital Regional de Presidente Prudente, contendo indivíduos de ambos os sexos, com idade acima de 18 anos. A população estudada foi composta por 44 pacientes, variando entre 18 à 91 anos. 63\% do total dos entrevistados apresentaram risco nutricional, sendo que, os indivíduos do sexo masculino, apresentaram maior probabilidade de possuir ou desenvolver riscos nutricionais durante o período de hospitalização em relação aos indivíduos do sexo feminino. Demonstrou-se a importância da intervenção nutricional imediatamente na admissão do paciente no serviço de emergência, reduzindo assim, a permanência hospitalar e custos da própria instituição com os tratamentos.

Palavras-chave: Desnutrição, Enfermos, Hospitalização, Desnutrição proteico-calórica, Sistema único de saúde .

\section{EVALUATION OF THE NUTRITIONAL STATUS OF PATIENTS ADMITTED TO THE RECEPTION AND RISK CLASSIFICATION SERVICE AT PRESIDENTE PRUDENTE REGIONAL HOSPITAL - SP}

\begin{abstract}
Malnutrition in a hospital environment is a public health problem that is associated with a significant increase in morbidity and mortality. The objective of this study was to evaluate the nutritional status through nutritional screening tool, adapted to the Malnutrition Screening Tool (MST). This was a field study, which was carried out at the Regional Hospital of Presidente Prudente, containing individuals of both sexes, aged over 18 years. The study population consisted of 44 patients, ranging from 18 to 91 years old. $63 \%$ of the total of the interviewees presented a nutritional risk, and the male subjects were more likely to have or develop nutritional risks during the hospitalization period in relation to the female subjects. The importance of nutritional intervention was immediately demonstrated in the admission of the patient to the emergency department, thus reducing hospital stay and the institution's own costs with the treatments.

Keywords: Malnutrition, Patients, Hospitalization, Protein-energy malnutrition, Unified health system.
\end{abstract}




\section{INTRODUÇÃO}

A desnutrição em ambiente hospitalar é um problema de saúde pública que está associada ao aumento significativo de morbidade e mortalidade ${ }^{1}$. É fato de que pacientes desnutridos apresentam maior tempo de hospitalização, o que tem relação direta com o aumento dos custos com tratamentos, se comparados aos indivíduos bem nutridos ${ }^{2}$.

No intuito de prevenir a desnutrição, é de fundamental importância a identificação do paciente em risco nutricional, por meio da triagem, para que se possa realizar intervenções oportunas, visando impedir ou retardar a desnutrição e suas consequências ${ }^{3}$.

A aplicação da triagem e avaliação nutricional se tornou importante para a evolução do paciente, além de ser uma ferramente reconhecida pelo Ministério da Saúde, no Brasil. É obrigatória na elaboração de protocolos para pacientes internados pelo Sistema Único de Saúde (SUS), como um instrumento que auxilia na determinação de custos para a terapia nutricional enteral e parenteral ${ }^{4}$.

A triagem nutricional é uma ferramenta utilizada a fim de facilitar a indentificação do risco nutricional pelos profissionais, objetivando a realização de planos de cuidado e intervenções precoces. Sua aplicação deve ter início em até 24 horas da admissão do paciente em nível hospitalar, e na primeira consulta em nível ambulatorial e domiciliar, a fim de identificar precocemente os pacientes que poderão se beneficiar da terapia nutricional ${ }^{5}$.

Não existe um instrumento considerado padrão-ouro para a avaliação do estado nutricional do paciente hospitalizado. A síntese de dados disponíveis, possibilita esse diagnóstico, e pode ser obtida por vários métodos tradicionais como antropometria, perda de peso, avaliação de sinais clínicos indicativos de desnutrição, alterações de exames bioquímicos, que detectam redução nas taxas de proteínas plasmáticas e de células mediadoras da imunidade, além de avaliação do consumo alimentar ${ }^{6}$.

O estado de hipercatabolismo provocado por algumas doenças; o longo período de hospitalização; idade superior a 60 anos; perda de peso recente e involuntária; modificações na consistência da dieta; alterações gastrointestinais, como náuseas, disfagia, dor abdominal e anorexia, prejudicam a ingestão alimentar, favorecendo a piora do estado nutricional e consequentemente, interferem negativamente na recuperação do paciente internado ${ }^{7,8}$.

Em pacientes gravemente doentes e nutricionalmente comprometidos, a produção de citocinas relacionadas ao stress (fator de necrose tumoral, interleucinas e interferon) e o hipercatabolismo proteico, levam à perda secundária de massa magra, principalmente do músculo esquelético, o qual se relaciona proporcionalmente com o aumento da morbidade e mortalidade 9 .

Em alguns casos, podem ocorrer dificuldades na identificação imediata da desnutrição no primeiro contato com o paciente, especialmente quando há a supressão de informações sobre o seu estado nutricional anterior ${ }^{10}$.

Desse modo, tornou-se importante identificar antecipadamente o paciente em risco nutricional admitido no Pronto Socorro, para que se possa, precocemente, realizar uma adequada intervenção nutricional, visando sua rápida recuperação, proporcionando um menor tempo de permanência hospitalar, possibilitando a redução de custos relacionados à internação. O objetivo do presente estudo, foi avaliar o estado nutricional dos pacientes admitidos no Pronto Socorro, por meio da realização de triagem nutricional, a fim de constatar a perda ponderal nos pacientes recém-admitidos, corrigir e previnir riscos nutricionais.

\section{MÉTOdOS}

Os procedimentos adotados, obedecem aos Critérios da Ética em Pesquisa com Seres Humanos conforme Resolução $n^{\circ}$. 466/2012 do Conselho Nacional de Saúde. A pesquisa teve início após a aprovação do Comitê de Ética em Pesquisa da Universidade do Oeste Paulista (UNOESTE) e foi aprovada com o número de protocolo 3219. 
Os participantes assinaram um termo de consentimento livre e esclarecido (TCLE) redigido para este fim, concordando em participar da pesquisa. Neste TCLE, foram informados sobre os objetivos do trabalho e os procedimentos aos quais foram expostos.

O presente projeto foi desenvolvido durante os meses de junho e julho de 2016, na área de acolhimento do Pronto Socorro do Hospital Regional de Presidente Prudente, SP, durante três dias da semana, nos horários entre $07 \mathrm{~h} 30$ a $11 \mathrm{~h} 30$.

Tratou-se de um estudo observacional quantitativo, no qual a população alvo foi escolhida por conveniência de forma aleatória e não probabilística. Foram convidados a participar da pesquisa os pacientes que se encontravam no serviço de acolhimento do Pronto Socorro no período do estudo, os quais foram submetidos à triagem nutricional (ANEXO 1) uma única vez após a avaliação feita pela enfermagem local, o qual classificava os pacientes de acordo com a complexidade de atendimento.

A triagem nutricional é um instrumento validado, composto por avaliação objetiva (Peso, Altura) e subjetiva, que identifica e avalia a perda de peso do paciente. Para tal, utilizou-se o instrumento de avaliação "Malnutrition screening tool" (MST), classificado como um instrumento breve, direto, validado, eficiente e fácil de ser aplicado no grande volume de pacientes que internam todos os dias nos hospitais. Além disso, tornou-se uma via de rastreamento sensível para identificar os pacientes em risco nutricional, levando de 3 a 5 minutos para ser preenchido e interpretado, pois abrange dados subjetivos, como história de perda de peso, perda de apetite, e alteração da ingestão alimentar, apresentando boa correlação com outros recursos mais demorados, complexos e que necessitam de dados antropométricos mais específicos do paciente ${ }^{11}$.

Para mensurar o peso e a altura do paciente, foi utilizada uma balança tipo "plataforma", com capacidade máxima de $150 \mathrm{~kg}$ e sensibilidade de 0,1 kg da marca Camry. A aferição da altura foi feita com a régua antropométrica de $200 \mathrm{~cm}$ acoplada à balança.

Foram incluídos no estudo os pacientes pertencentes à triagem hospitalar, conscientes, em estado de alerta, não confusos e aptos a responder ao questionário, além de ter condições de ser submetidos à avaliação nutricional (deambulando), de qualquer raça ou sexo e idade mínima de 18 anos.

Foram excluídos da amostra os pacientes que não atendiam aos requisitos citados, assim como os classificados, pela enfermagem, como "graves", os quais eram encaminhados diretamente à sala de emergência para internação.

Os dados foram agrupados e armazenados em planilha, utilizando o programa Microsoft Office Exel $^{\oplus}$ e apresentados em forma de tabelas e gráficos de setores para melhor visualização.

\section{RESULTADOS:}

A população estudada foi composta por 44 pacientes, sendo estes, 26 do sexo masculino e 18 do sexo feminino, variando entre 18 a 91 anos. Todos os pacientes possuíam encaminhamento para consulta ou internação neste período. 


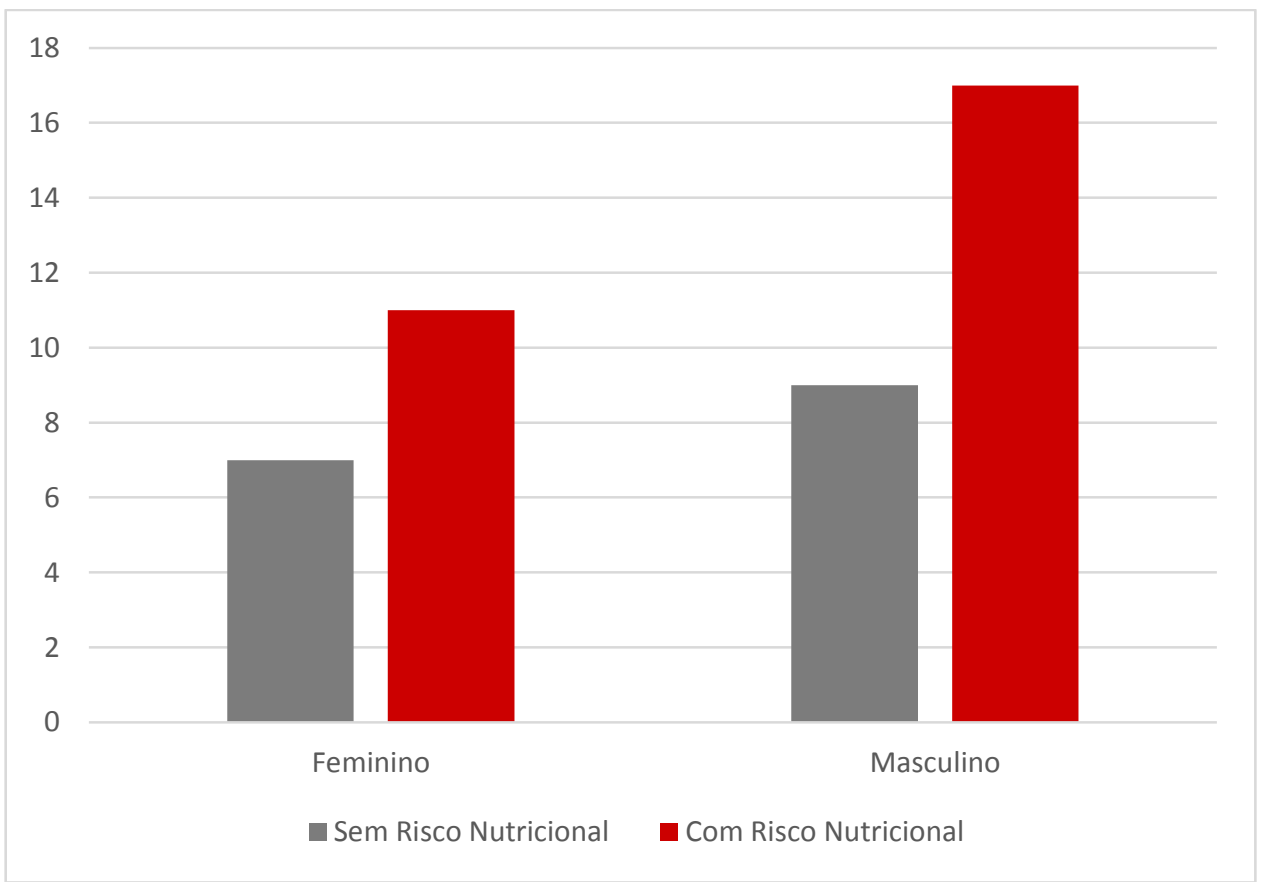

Figura 1. Gráfico amostral de casos de risco nutricional entre mulheres e homens.

A figura 1 apresenta a relação entre induvíduos do sexo masculino e feminino envolvidos na pesquisa, com relação ao risco nutricional apresentado. Observou-se que no período do estudo, houve uma maior procura da população masculina pelos serviços oferecidos no prontosocorro.

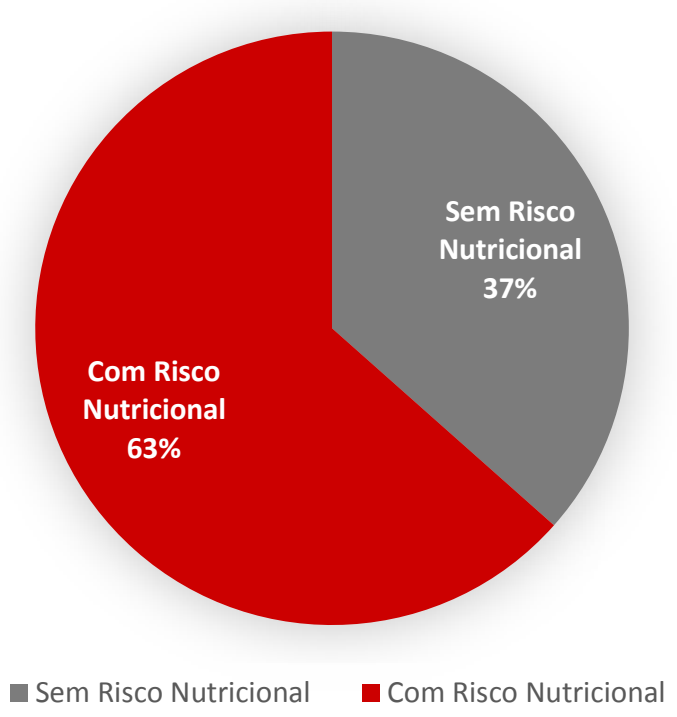

Figura 2. Percentuais referentes ao risco nutricional apresentado pelos pacientes.

A figura 2 apresenta os resultados da pesquisa realizada em relação a presença ou não de risco nutricional. Segundo os resultados, 63\% do total dos indivíduos apresentaram risco nutricional, sendo que, de acordo com a figura 1 , os indivíduos do sexo masculino apresentaram maior probabilidade de possuir ou desenvolver riscos nutricionais durante o período de hospitalização em relação a indivíduos do sexo feminino.

\section{DISCUSSÃO}

Para maior segurança e facilidade na aplicação das ferramentas de diagnóstico nutricional, torna-se necessário a utilização de protocolos nas instituições de saúde para a padronização e 
descrição das ferramentas utilizadas, a fim de evitar limitações no uso das mesmas.

Especialistas da área da saúde deparam-se com dificuldades para utilizar a maioria das ferramentas atualmente validadas para avaliação nutricional, devido à limitação de tempo, reprodutibilidade do método, organização ou custos ${ }^{12}$.

A prevalência do risco nutricional detectada, neste trabalho, foi de $63,7 \%$. Valor superior ao do estudo realizado em 2009, por meio do instrumento "Nutritional Risk Screening, 2002", em um hospital de Belo Horizonte $-\mathrm{MG}^{13}$.

Os indivíduos do sexo masculino, apresentaram maiores índices de risco nutricional $(65,3 \%)$.

Segundo um estudo realizado no Sul do Brasil, há falta de envolvimento em ações voltadas à promoção da saúde e prevenção de doenças pelos homens, sendo que eles possuem maior dificuldade em se reconhecerem doentes, além de terem receio pela descoberta de alguma doença grave, fazendo com que, procurem com menor frequência os serviços de saúde, prejudicando o prognóstico das doenças ${ }^{14}$.

No setor de emergência do Hospital Regional de Presidente Prudente, a partir do referenciamento de pacientes, observou-se um aumento do fluxo dos casos graves, necessitando na maioria das vezes, de cuidados intensivos.

Um fator que pode influenciar na aceitação da alimentação pelos pacientes, é a presença de dor, sendo que a dor sem manejo adequado, faz o paciente privar-se da alimentação pelo desconforto que a ingestão alimentar pode promover ou exacerbar. Dessa forma, a redução na ingestão alimentar contribui para a perda de peso, que pode também associar-se com a redução da capacidade funcional ${ }^{15}$.

Respostas metabólicas graves ocasionadas por hipermetabolismo e hipercatabolismo proteico, são frequentes em pacientes graves, tornando-os mais vulneráveis a desenvolver um quadro de desnutrição. Nesses casos, a desnutrição é relacionada a vários fatores, como a anorexia, perda de aminoácidos, uso de medicamentos que interferem na absorção de nutrientes, distúrbios hormonais e gastrointestinais, levando o indivíduo a um pior prognóstico, devendo ser detectada e prevenida o mais precocemente possível, a fim de retardar ou inclusive, eliminar o risco de morbidade e mortalidade ${ }^{16,17}$.

Transtornos com relação à nutrição e imunidade como consequência de processo infeccioso, podem acarretar desnutrição por meio de infecções intestinais, ocasionando alterações na absorção e biodisponibilidade de nutrientes; de processo febril que pode causar aumento da demanda energética e de infecções crônicas que intensificam a glicogênese e a lipogênese, alterando o metabolismo de carboidratos, lipídios, proteínas, níveis de micronutrientes e balanço hidroeletrolítico, além de alterações hormonais ${ }^{18}$.

Portanto é de extrema importância que se faça o diagnóstico nutricional imediatamente na admissão do paciente nas unidades de emergência, sendo imprescindível a presença do nutricionista no setor de emergência de um hospital, para que se possa realizar, na admissão do paciente, avaliação nutricional, utilizando-se de ferramentas adequadas, a fim de detectar carências nutricionais e dessa forma, reduzir os índices de desnutrição intra-hospitalar, tempo de permanência nos hospitais e redução de custos com a hospitalização.

\section{REFERÊNCIAS}

1. Raslan M, Gonzalez MC, Dias MCG, Paes-Barbosa FC, Cecconello I, Waitzberg DL. Aplicabilidade dos métodos de triagem nutricional no paciente hospitalizado. Rev. Nutr. 2008; 21(5): 553-561. DOI: http://dx.doi.org/10.1590/S1415-52732008000500008

2. Szuck P, Führ LM, Garcia MF, Da Silva AT, Wazlawik E. Association between nutritional indicators and risk of hospitalization among hemodialysis patients. Rev. Nutr. 2016; 29 (3): 317- 


\section{DOI: http://dx.doi.org/10.1590/1678-98652016000300002}

3. Teixeira AF, Viana KDAL. Nutritional screening in hospitalized pediatric patients: a systematic review. J. Pediatr. 2016; 92(4): 343-352. DOI: 10.1016/j.jped.2015.08.011

4. Dias MCG, Van ADPJ, Catalani LA, Rey JSF, Gonzales MC, Coppini L. et al. Triagem e Avaliação do Estado Nutricional. In: Projeto Diretrizes. AMB. 2011. 16 p.

5. Fidelix MSP. Sistematização do cuidado de nutrição. In: manual orientativo. ASBRAN. 2014. $68 \mathrm{p}$.

6. Aquino R de C, Philippi ST. Desenvolvimento e avaliação de instrumentos de triagem nutricional. Rev. bras. enferm. 2012;65 (4): 607-613. DOI: http://dx.doi.org/10.1590/S003471672012000400009

7. Fragas RFM, De Oliveira MC. Risk factors associated with malnutrition in hospitalized patients. Rev. Nutr. 2016; 29 (3): 329-336. DOI: http://dx.doi.org/10.1590/1678$\underline{98652016000300003}$

8. Martins C. Introdução à Avaliação do Estado Nutricional. In: Inst. cristina martins. 2011. 16 p.

9. González CBG, Monares ZE, Jiménez CAV. Predicción del catabolismo proteico sin utilizar nitrógeno urinario excretado de 24 horas en el paciente críticamente enfermo. Rev. Asoc. Mex. Med. Crít. Ter. Intensiva. 2015; 29 (1): 8-12. DOI: http://www.scielo.org.mx/scielo.php?script=sci_arttext\&pid=S0187-84332015000100002\&lng=es

10. Teixeira AC de C, Caruso L, Soriano FG. Terapia nutricional enteral em unidade de terapia intensiva: infusão versus necessidades. Rev. bras. ter. intensiva. 2006;18 (4): 331-337. DOI: http://dx.doi.org/10.1590/S0103-507X2006000400003

11. Garcia RS, Tavares LR da C, Pastore CA. Nutritional screening in surgical patients of a teaching hospital from Southern Brazil: the impact of nutritional risk in clinical outcomes. Einstein (São Paulo). 2013; 11 (2):147-152. DOI: http://dx.doi.org/10.1590/S1679-45082013000200002

12. Jansen AK, Silveira ALM, De Oliveira MAB, Pimenta AM. Desfecho Terapêutico de Pacientes em Risco Nutricional Admitidos em um Hospital Universitário. Rev. min. enferm. 2013; 17 (3): 658664. DOI: $10.5935 / 1415-2762.20130048$

13. De Jesus MCP, Santos SM dos R, Lamas JLT, De Jesus PBR, Gonçalves P da L de C, Jory MV. Marcadores de saúde do homem em um município de pequeno porte. Esc. Anna Nery. 2014; 18 (4): 650-655. DOI: 10.5935/1414-8145.20140092

14. Poziomyck AK, Fruchtenicht AVG, Kabke GB, Volkweis BS, Antoniazzi JL, Moreira LF. Reliability of nutritional assessment in patients with gastrointestinal tumors. Rev. Col. Bras. Cir. 2016; 43 (3): 189-197. DOI: 10.1590/0100-69912016003006

15. Silva PB, Trindade LCT, Gallucci MC, Schirr RA. Prevalência de desnutrição e dor em pacientes admitidos pelo serviço de triagem em hospital oncológico. Rev. dor. 2013; 14 (4): 263- 
266. DOI: http://dx.doi.org/10.1590/S1806-00132013000400006

16. Fruchtenicht AVG, Poziomyck AK, Kabke GB, Loss SH, Antoniazzi JL, Steemburgo T. et al . Avaliação do risco nutricional em pacientes oncológicos graves: revisão sistemática. Rev. bras. ter. intensiva. 2015; 27 (3): 274-283. DOI: http://dx.doi.org/10.5935/0103-507X.20150032

17. Genoni CA, Togawa KN, Lima LP, Fagiani M de AB, Genaro SC. Estado nutricional de pacientes com doença renal crônica em tratamento dialítico. Rev. saber acad. 2015; 1 (19): 50-62. DOI: http://uniesp.provisorio.ws/revista/revista19/pdf/artigos/05.pdf

18. Ferraz $L$ de $F$, Viriato $A$, Moura $A$ de. Análise do diagnóstico nutricional de pacientes em assistência hospitalar de infectologia. O mundo da saúde. 2013; 37 (3): 253-258. DOI: http://www.saocamilo-sp.br/pdf/mundo_saude/106/1821.pdf 
Anexo 1 - Triagem Nutricional

Data da Triagem:

Paciente:

Peso Habitual:

(kg) Peso Atual:

(kg) Altura:

$(\mathrm{cm})$

Você perdeu peso recentemente de forma involuntária?

\begin{tabular}{|l|l|}
\hline ( ) Não & 0 pontos \\
\hline ( ) Não sei & 2 pontos \\
\hline \multicolumn{2}{|l|}{ Se SIM, quantos quilos você perdeu? } \\
\hline ( ) $1-5$ & 1 ponto \\
( ) 6 a 10 & 2 pontos \\
( ) $11-15$ & 3 pontos \\
( ) $>15$ & 4 pontos \\
( ) Não sei & 2 pontos
\end{tabular}

Você tem se alimentado mal por sentir menos apetite?

( ) Não 0 pontos

( ) Sim 1 ponto

Total de Pontos

Fonte: Malnutrition Screening Tool (MST)

Classificação:

$0-1$ : Sem risco nutricional

2 - 4: Em risco nutricional 